Pacific

Journal of

Mathematics

SOME PROPERTIES OF SQUEEZING FUNCTIONS ON BOUNDED DOMAINS

Fusheng Deng, Qian GuAN AND LiYou Zhang

Volume $257 \quad$ No. 2

June 2012 


\title{
SOME PROPERTIES OF SQUEEZING FUNCTIONS ON BOUNDED DOMAINS
}

\author{
FUSHENG DENG, QIAN GUAN AND LIYOU ZHANG
}

\begin{abstract}
This paper introduces the notion of squeezing functions on bounded domains and studies some of their properties. The relation to geometric and analytic structures of bounded domains will be investigated. Existence of related extremal maps and continuity of squeezing functions are proved. Holomorphic homogeneous regular domains introduced by Liu, Sun and Yau are exactly domains whose squeezing functions have positive lower bounds. Completeness of certain intrinsic metrics and pseudoconvexity of holomorphic homogeneous regular domains are proved by alternative method. In the dimension one case, we get a neat description of boundary behavior of squeezing functions of finitely connected planar domains. This leads to necessary and sufficient conditions for a finitely connected planar domain to be a holomorphic homogeneous regular domain. Consequently, we can recover some important results in complex analysis. For annuli, we obtain some interesting properties of their squeezing functions. Finally, we present some examples of bounded domains whose squeezing functions can be given explicitly.
\end{abstract}

\section{Introduction}

Bounded domains are elementary objects of study in complex analysis. To study complex and geometric structures of bounded domains, one may consider holomorphic maps from bounded domains to some standard domains such as balls and vice versa. The basic idea goes back to Carathéodory, and a typical example is the definitions of the Carathéodory metric and the Kobayashi metric. Holomorphic maps from bounded domains to the unit ball with certain extremal properties are called Carathéodory maps, which can be explicitly given for some special domains such as bounded symmetric domains [Kubota 1981; 1982a; 1982b; 1983] and ellipsoids [Ma 1997]. Recently, by considering embeddings of general bounded domains into the unit ball, a new concept of holomorphic homogeneous regular domains

This research was partially supported by NSFC grants (10901152 and 11001148) and the president fund of GUCAS..

MSC2010: 32F45, 32H02.

Keywords: squeezing function, extremal map, holomorphic homogeneous regular domain. 
was introduced [Liu et al. 2004; Liu et al. 2005]. Holomorphic homogeneous regular domains are generalizations of Teichmüller spaces, and they admit some nice geometric and analytic properties [Liu et al. 2004; Liu et al. 2005; Yeung 2009].

Motivated by the above works, especially [Liu et al. 2004; Liu et al. 2005], we introduce the notion of squeezing functions defined on general bounded domains as follows:

Definition 1.1. Let $D$ be a bounded domain in $\mathbb{C}^{n}$. For $p \in D$ and an (open) holomorphic embedding $f: D \rightarrow B^{n}$ with $f(p)=0$, we define

$$
s_{D}(p, f)=\sup \left\{r \mid B^{n}(0, r) \subset f(D)\right\},
$$

and the squeezing number $s_{D}(p)$ of $D$ at $p$ is defined as

$$
s_{D}(p)=\sup _{f}\left\{s_{D}(p, f)\right\},
$$

where the supremum is taken over all holomorphic embeddings $f: D \rightarrow B^{n}$ with $f(p)=0, B^{n}$ representing the unit ball in $\mathbb{C}^{n}$, and $B^{n}(0, r)$ representing the ball in $\mathbb{C}^{n}$ with center 0 and radius $r$. We call $s_{D}$ the squeezing function on $D$.

By definition, it is clear that squeezing functions are invariant under biholomorphic transformations, so a squeezing function can be viewed as a kind of holomorphic invariant of a bounded domain. The main purpose of the present paper is to investigate some properties of squeezing functions and their relations with geometric and analytic structures of bounded domains.

Squeezing functions are always positive and bounded above by 1 . It is interesting to estimate their lower and upper bounds, which are numerical holomorphic invariants of bounded domains, by the holomorphic invariance of squeezing functions. Holomorphic homogeneous regular domains defined in [Liu et al. 2004; Liu et al. 2005] are exactly bounded domains whose squeezing functions admit positive lower bounds. They contain some interesting objects such as bounded homogeneous domains, Teichmüller spaces, bounded domains covering compact Kähler manifolds, and strictly convex domains with $C^{2}$-boundary [Yeung 2009].

It is easy to see that the squeezing function of the unit ball $B^{n}$ is constant with value 1. A natural question is whether the squeezing function of a bounded domain $D$ can attain the value 1 at some point $x$ in $D$ if $D$ is not holomorphic equivalent to $B^{n}$. This question is related to the existence of an extremal map which realizes the supremum $s_{D}(x)$. In other words, the existence of a holomorphic embedding $f: D \rightarrow B^{n}$ such that $f(x)=0$ and $B^{n}\left(0, s_{D}(x)\right) \subset f(D)$. We will prove the existence of extremal maps by using a higher dimensional generalization of the Hurwitz theorem which we will also prove. As a consequence, $S_{D}$ attains the value 1 in $D$ if and only if $D$ is holomorphic equivalent to the unit ball. On the 
other hand, as we will see, there exist domains whose squeezing functions have supremum 1, but are not holomorphic equivalent to the unit ball.

An elementary property of regularity of squeezing functions is their continuity. This can be proved by using the decreasing property of Kobayashi metrics. From the continuity property, one can see that a bounded domain is a holomorphic homogeneous regular domain if it covers a compact complex manifold.

Properties of squeezing functions are able to reflect some geometric and analytic properties of bounded domains. The boundary behavior of squeezing functions implies a certain boundary estimate of the Carathéodory metric, which implies completeness of the metric in some special cases. For a bounded domain whose squeezing function admits a positive lower bound, that is, a holomorphic homogeneous regular domain, it is known that the intrinsic metrics - the Carathéodory metric, the Kobayashi metric, and the Bergman metric — on it are equivalent [Liu et al. 2004; Liu et al. 2005], and they are all complete [Yeung 2009]. We will prove the completeness of these metrics by an alternative method based on Look's result [1958] on comparing the Bergman metric and the Carathéodory metric. A result in several complex variables states that completeness of the Carathéodory metric of a domain implies its pseudoconvexity [Jarnicki and Pflug 1993], and hence a holomorphic homogeneous regular domain must be a pseudoconvex domain.

Squeezing functions of planar domains have nice properties. For finitely connected planar domains, we get a neat description of the boundary behavior of their squeezing functions. As a result, we get the necessary and sufficient condition for such a domain to be a holomorphic homogeneous regular domain. Surprisingly, the squeezing function $s_{D}$ of a bounded planar domain $D$ with smooth boundary admits the boundary behavior

$$
\lim _{z \rightarrow \partial D} s_{D}(z)=1
$$

By the continuity of squeezing functions, equality (1) implies that all smoothly bounded planar domains are holomorphic homogeneous regular domains. As a result, we can recover some important results about planar domains. For example, the three intrinsic metrics mentioned above on a bounded planar domain with smooth boundary are all complete, and they are equivalent. Also, a smoothly bounded planar domain must be hyperconvex, that is, it admits a bounded exhaustive subharmonic function. In particular, equality (1) also implies that $s_{D}$ can be extended continuously to $\bar{D}$ for a planar domain $D$ with smooth boundary. We don't know whether this is true in the general case, that is, whether $s_{D}$ can be extended continuously to $\bar{D}$ for all bounded domains $D \subset \mathbb{C}^{n}$ with smooth boundary.

It is clear that the product of two holomorphic homogeneous regular domains is still a holomorphic homogeneous regular domain, so smoothly bounded planar 
domains and their products provide a class of holomorphic homogeneous regular domains which are generally not contained in the list of holomorphic homogeneous regular domains mentioned above. As remarked in [Liu et al. 2004; Liu et al. 2005], it may be interesting to investigate whether the Kobayashi metric and the Carathéodory metric on a Teichmüller space coincide or not; on the other hand, the Kobayashi metric and the Carathéodory metric on general holomorphic homogeneous regular domains constructed here don't coincide.

The simplest nontrivial smoothly bounded planar domains are annuli. However, even in this special case, squeezing functions admit nontrivial properties. With some investigation, we conjecture that the conformal structure of an annulus is characterized by the exact lower bound of its squeezing function.

The squeezing functions can be given explicitly for classical bounded symmetric domains. In this special case, we see that the extremal maps for squeezing functions defined as above can be given by the Carathéodory maps (see Section 7 for exact definition). However, this does not hold for general domains such as annuli. In fact, the Carathéodory maps (often called Ahlfors maps for planar domains) of a bounded planar domain can not even be injective if the domain is not simply connected [Fisher 1983]. It seems that the obstruction for the coincidence of the two types of extremal maps comes from topology. Therefore, we conjecture that the extremal maps of a contractible domain are given by Carathéodory maps.

The rest of this article is organized as follows. In Section 2, we generalize the Hurwitz theorem from one complex variable analysis to several complex variables, and use this generalization to establish the existence of extremal maps that are defined as above. In Section 3, we prove the continuity of squeezing functions of general bounded domains. In Section 4, we give a boundary estimate of Carathéodory metrics in term of boundary behavior of squeezing functions, and prove the completeness of certain intrinsic metrics on holomorphic homogeneous regular domains. As a corollary, we get the pseudoconvexity of these domains. In Section 5, we study squeezing functions on planar domains and prove equality (1) of smoothly bounded planar domains. We also construct a class of planar holomorphic homogeneous regular domains which are infinitely connected. In Section 6, we focus on squeezing functions on annuli, and in Section 7, we give some examples of bounded domains whose squeezing functions can be given explicitly.

\section{Generalized Hurwitz theorem and the existence of extremal functions}

The main aim of this section is to establish the existence of extremal maps related to squeezing functions.

Theorem 2.1. Let $D$ be a bounded domain in $\mathbb{C}^{n}$. Then for any $x \in D$, there exists a holomorphic embedding $f: D \rightarrow B^{n}$ such that $f(x)=0$ and $B^{n}\left(0, s_{D}(x)\right) \subset f(D)$. 
By definition we have $s_{D}(z) \leq 1$ for all $z \in D$. By Theorem 2.1, we see that $s_{D}(z)=1$ for some $z \in D$ if and only if $D$ is holomorphically equivalent to $B^{n}$.

To prove Theorem 2.1, we need to generalize Hurwitz's theorem in classical complex analysis to several complex variables. Hurwitz's theorem in one complex variable says that the limit of a sequence of univalent functions on a planar domain is univalent unless it is constant [Remmert 1998]. Of course there is no direct generalization of this result in higher dimensions. However, a modified version described in the following theorem still holds.

Theorem 2.2. Let $D$ be a bounded domain in $\mathbb{C}^{n}$ and $x \in D$. Let $f_{i}$ be a sequence of injective holomorphic maps from $D$ to $\mathbb{C}^{n}$ such that $f_{i}(x)=0 \in \mathbb{C}^{n}$ for all $i$. Suppose $f_{i}$ converges to a map $f: D \rightarrow \mathbb{C}^{n}$ uniformly on compact subsets of $D$. If there exists a neighborhood $U$ of 0 in $\mathbb{C}^{n}$ such that $U \subset f_{i}(D)$ for all $i$, then $f$ is injective.

To prove Theorem 2.2, we need two lemmas.

Lemma 2.3. Let $D$ be a domain in $\mathbb{C}^{n}$ and $\varphi_{i}$ a sequence of holomorphic functions which is convergent to $\varphi: D \rightarrow \mathbb{C}$ uniformly on compact subsets of $D$. If all $\varphi_{i}$ have no zero in $D$, then $\varphi$ has no zero in $D$ unless it is identically zero.

Proof. By the identity theorem of holomorphic functions, we may assume $D$ is a ball. Assume there exists $x \in D$ such that $\varphi(x)=0$. For any point $z \in D$, consider the intersection of $D$ and the complex line containing $x$ and $z$. Then a version of the classical Hurwitz theorem [Remmert 1998, Corollary, pg. 162] implies that $\varphi(z)=0$, so $\varphi$ is identically zero on $D$.

The second lemma we need in the proof of Theorem 2.2 is the generalized Rouché's theorem in higher dimensions, whose proof relies on the mapping degree theory in differential topology.

Lemma 2.4 [Lloyd 1979, Theorem 3]. Let D be a bounded domain in $\mathbb{C}^{n}$. Suppose $f$ and $g$ are two holomorphic maps from $D$ to $\mathbb{C}^{n}$ such that

$$
\|g(z)\|<\|f(z)\|, \quad z \in \partial D .
$$

Then $f$ and $f+g$ have the same number of zeros in D, counting multiplicities, where $\|\cdot\|$ is the standard norm in $\mathbb{C}^{n}$.

Proof of Theorem 2.2. Let $g_{i}=\left.f_{i}^{-1}\right|_{U}$. By Montel's theorem, the sequence $\left\{g_{i}\right\}$ is convergent to a holomorphic map $g: U \rightarrow \mathbb{C}^{n}$ uniformly on compact subsets of $U$. Noting that $g(0)=x$ is an interior point of $D$, we can assume $g(U) \subset D$ by taking $U$ small enough. It is clear that $f_{i} g_{i}=\operatorname{Id}_{U}$ for all $i$. Letting $i$ tend to $\infty$, we get $f g=\operatorname{Id}_{U}$. This implies that the Jacobian determinant det $J_{f}(x)$ of $f$ at $x$ is not zero. Since the $f_{i}$ are all injective, $\operatorname{det} J_{f_{i}}(z) \neq 0$ for all $i$ and all $z \in D$ [Fritzsche and Grauert 2002, Theorem 8.5]. Note that $\operatorname{det} J_{f_{i}}$ converges to $\operatorname{det} J_{f}$ 
uniformly on compact subsets of $D$. By Lemma 2.3 , we see that det $J_{f}(z) \neq 0$ for all $z \in D$. We prove $f$ is injective. If it is not the case, there exist $z_{1}, z_{2} \in D$, $z_{1} \neq z_{2}$, such that $f\left(z_{1}\right)=f\left(z_{2}\right)$. Since det $J_{f} \neq 0$, we can choose a neighborhood $\Omega \subset \subset D$ of $z_{1}$ such that $\left.f\right|_{\bar{\Omega}}$ is injective and $z_{2} \notin \bar{\Omega}$. Set $\tilde{f}_{i}=f_{i}-f_{i}\left(z_{2}\right)$ and $\tilde{f}=f-f\left(z_{2}\right)$. Then $\tilde{f}_{i}$ converges to $\tilde{f}$ uniformly on $\bar{\Omega}$. Note that $\tilde{f}$ has a zero in $\Omega$ and $\tilde{f}^{-1}(0) \cap \partial \Omega=\varnothing$. By Lemma $2.4, \tilde{f}_{i}$ has a zero in $\Omega$ for $i$ large enough, which contradicts the fact that the $f_{i}$ are all injective on $D$.

Remark 2.5. The assumptions in Theorem 2.2 that $D$ is bounded and all $f_{i}(D)$ contain a fixed neighborhood of $0 \in \mathbb{C}^{n}$ are necessary. In fact, without either assumption, the result in Theorem 2.2 does not hold. For example, taking

$$
f_{i}\left(z_{1}, z_{2}\right)=\left(z_{1}, z_{2} / i\right)
$$

as a sequence of holomorphic maps from $D=\mathbb{C}^{2}$ to itself, the limit map $f: D \rightarrow$ $\mathbb{C}^{2}$ is given by $f\left(z_{1}, z_{2}\right)=\left(z_{1}, 0\right)$. It is not injective even if the $f_{i}$ are injective and $\mathbb{C}^{2} \subset f_{i}(D)$ for all $i$; the restrictions on $\left.f_{i}\right|_{B^{2}}$ give a sequence of injective holomorphic maps from $B^{2}$ to $\mathbb{C}^{2}$ with limit map $\left.f\right|_{B^{2}}$, which is not injective since not all $f_{i}\left(B^{2}\right)$ contain a fixed neighborhood of $0 \in \mathbb{C}^{2}$. On the other hand, by a result in [Hahn 1976], the two assumptions can be replaced by assuming that $\left|\operatorname{det} J_{f_{i}}(x)\right|$ have a positive lower bound.

Proof of Theorem 2.1. By the definition of squeezing functions, there exist a sequence of holomorphic embeddings $f_{i}: D \rightarrow B^{n}$ with $f_{i}(x)=0$, and a sequence of increasing positive numbers $r_{i}$ convergent to $s_{D}(x)$ such that $B^{n}\left(0, r_{i}\right) \subset f_{i}(D)$. By Montel's theorem, there exists a subsequence $\left\{f_{i_{k}}\right\}$ of $f_{i}$ which converges to a homomorphic map $f: D \rightarrow \mathbb{C}^{n}$ uniformly on compact subsets of $D$. Since $B^{n}\left(0, r_{1}\right) \subset f_{i}(D)$ for all $i$, by Theorem $2.2, f$ is injective. In particular it is an open map. Hence $f(D) \subset B^{n}$. Then we get a holomorphic embedding $f: D \rightarrow B^{n}$ with $f(x)=0$.

To prove $B^{n}\left(0, s_{D}(x)\right) \subset f(D)$, it suffices to prove $B^{n}\left(0, r_{j}\right) \subset f(D)$ for each fixed integer $j$. By assumption, $B^{n}\left(0, r_{j}\right) \subset f_{i}(D)$ for all $i>j$. Let $g_{i}=$ $\left.f_{i}^{-1}\right|_{B^{n}\left(0, r_{j}\right)}$. Then we have $f_{i_{k}} g_{i_{k}}=\operatorname{Id}_{B^{n}\left(0, r_{j}\right)}$ for $i_{k}>j$. By Montel's theorem, we may assume that the sequence $\left\{g_{i_{k}}\right\}$ converges to a holomorphic map $g: B^{n}\left(0, r_{j}\right) \rightarrow \mathbb{C}^{n}$ uniformly on compact subsets of $B^{n}\left(0, r_{j}\right)$. We want to prove that $g\left(B^{n}\left(0, r_{j}\right)\right) \subset D$. Note that $g(0)=x$. Hence there exists a neighborhood $U$ of 0 in $B^{n}\left(0, r_{j}\right)$ such that $g(U) \subset D$. This implies $\left.f \cdot g\right|_{U}$ is defined, and it is clearly equal to the identity map $\operatorname{Id}_{U}$, so det $J_{g}(0) \neq 0$. Since det $J_{g_{i}} \neq 0$ for all $i>i_{0}$, by Lemma 2.3, we have $\operatorname{det} J_{f} \neq 0$ and hence $g$ is an open map, which implies that $g\left(B^{n}\left(0, r_{j}\right)\right) \subset D$. Therefore $f g: B^{n}\left(0, r_{j}\right) \rightarrow B^{n}\left(0, r_{j}\right)$ is a well defined map. It is clear that $f g=\operatorname{Id}_{B^{n}\left(0, r_{j}\right)}$, so we have $B^{n}\left(0, r_{j}\right) \subset f(D)$. 


\section{Continuity of squeezing functions}

In this section, we will prove that the squeezing function on any bounded domain is continuous. As a consequence, a bounded domain is a holomorphic homogeneous regular domain if it covers a compact complex manifold.

Theorem 3.1. The squeezing function $s_{D}$ of any bounded domain $D$ in $\mathbb{C}^{n}$ is continuous.

Proof. Since $D$ is a bounded domain, the Kobayashi metric on $D$ is nondegenerate.

Let $a$ be an arbitrary point in $D,\left\{z_{k}\right\}$ a sequence in $D$ convergent to $a$, and $\epsilon$ an arbitrary positive number. By Theorem 2.1, there exists a holomorphic embedding $f: D \rightarrow B^{n}$ such that $f(a)=0$ and $B^{n}\left(0, s_{D}(a)\right) \subset f(D)$. Since $f$ is continuous, there exists an integer $N$ such that $\left\|f\left(z_{k}\right)\right\|<\epsilon$ for $k>N$. Define $f_{k}: D \rightarrow \mathbb{C}^{n}$ as

$$
f_{k}(z)=\frac{f(z)-f\left(z_{k}\right)}{1+\epsilon}
$$

for $k>N$. Then $f_{k}(D) \subset B^{n}, f_{k}\left(z_{k}\right)=0$, and

$$
B^{n}\left(0, \frac{s_{D}(a)-\epsilon}{1+\epsilon}\right) \subset f_{k}(D) .
$$

This implies that $s_{D}\left(z_{k}\right) \geq\left(s_{D}(a)-\epsilon\right) /(1+\epsilon)$. Letting $\epsilon$ tend to 0 , we get

$$
\liminf _{k \rightarrow \infty} s_{D}\left(z_{k}\right) \geq s_{D}(a) .
$$

Let $K_{D}(\cdot, \cdot)$ be the Kobayashi distance on $D$. It is known that $K_{D}$ is continuous on $D \times D$ [Kobayashi 1998]. So we have $K_{D}\left(z_{k}, a\right) \rightarrow 0$ as $k \rightarrow \infty$. By Theorem 2.1, for each $k$, there exists a holomorphic embedding $f_{k}: D \rightarrow B^{n}$ such that $f_{k}\left(z_{k}\right)=0$ and $B^{n}\left(0, s_{D}\left(z_{k}\right)\right) \subset f_{k}(D)$. By the decreasing property of Kobayashi distances [Kobayashi 1998], we have

$$
K_{B^{n}}\left(f_{k}\left(z_{k}\right), f_{k}(a)\right)=K_{B^{n}}\left(0, f_{k}(a)\right) \leq K_{D}\left(z_{k}, a\right)
$$

for all $k$. So $K_{B^{n}}\left(0, f_{k}(a)\right) \rightarrow 0$, which implies that $f_{k}(a)$ tends to 0 in the ordinary topology [Barth 1972]. So, for any positive number $\epsilon$, there exists an integer $M$ such that $\left\|f_{k}(a)\right\|<\epsilon$ for $k>M$. This implies

$$
s_{D}(a) \geq \frac{s_{D}\left(z_{k}\right)-\epsilon}{1+\epsilon}, .
$$

so we have

$$
s_{D}(a) \geq \limsup _{k \rightarrow \infty} \frac{s_{D}\left(z_{k}\right)-\epsilon}{1+\epsilon} .
$$

Letting $\epsilon$ tend to 0 , we get

$$
s_{D}(a) \geq \limsup _{k \rightarrow \infty}\left(z_{k}\right) .
$$


So $\lim _{k \rightarrow \infty} s_{D}\left(z_{k}\right)=s_{D}(a)$, that is, $s_{D}$ is continuous at $a$. Note that $a$ is arbitrary, so $s_{D}$ is continuous on $D$.

For $r \in[0,1)$, we define

$$
\sigma(r)=\log \frac{1+r}{1-r} .
$$

It is clear that $\sigma(c)$ is strictly increasing for $0 \leq c<1$, and its inverse is given by $\sigma^{-1}(w)=\tanh (w / 2)$. For a point $z \in B^{n}$, the Kobayashi distance from 0 to $z$ is $\sigma(|z|)$. For two nonnegative numbers $u$ and $v$, it is not difficult to prove that

$$
\sigma^{-1}(u+v) \leq \sigma^{-1}(u)+\sigma^{-1}(v) .
$$

Let $D$ be a bounded domain in $\mathbb{C}^{n}$. We define a function $T(\cdot, \cdot)$ on $D \times D$ as

$$
T(x, y)=\sigma^{-1}\left(K_{D}(x, y)\right) .
$$

Then the above properties of $\sigma$ imply that $T(\cdot, \cdot)$ is a metric on $D$. Since $K_{D}$ induces the ordinary topology of $D$, so does $T$. From the proof of Theorem 3.1, one can directly get the following:

Theorem 3.2. The squeezing function $s_{D}$ of $D$ is Lipschitz continuous with respect to the metric $T$. In fact, we have

$$
\left|s_{D}(x)-s_{D}(y)\right| \leq 2 T(x, y), \quad x, y \in D .
$$

Remark 3.3. The same result as in the above theorem still holds if we replace Kobayashi distance in the definition of $T(\cdot, \cdot)$ by Carathéodory distance.

By Theorem 3.1, we directly get the following result, proved in [Yeung 2009]:

Corollary 3.4. Let $D$ be a bounded domain that covers a compact complex manifold. Then $D$ is a holomorphic homogeneous regular domain.

Proof. Let $X$ be a compact complex manifold that is covered by $D$. By the holomorphic invariance of squeezing functions, $s_{D}(z)$ can be pushed down to a function on $X$. By Theorem 3.1, $s_{D}(z)$ is continuous. Note that $s_{D}(z)$ is also positive, and it must attain a positive lower bound on $X$, and hence on $D$.

\section{Relations between intrinsic metrics and squeezing functions}

The main purpose of this section is to investigate relations between squeezing functions and some intrinsic metrics on bounded domains. We give a boundary estimate of the Carathéodory metric of a bounded planar domain in term of boundary behavior of its squeezing function. In fact, a similar but weaker form of this result still holds in a higher dimensional case. We then focus on bounded holomorphic homogeneous regular domains, and prove that the Carathéodory metric, the 
Kobayashi metric, and the Bergman metric on these domains are complete. As a result, a holomorphic homogeneous regular domain must be pseudoconvex.

We first need the following lemma, which is known as the Koebe quarter theorem in classical complex analysis.

Lemma 4.1 [Ahlfors 1973]. Let $\Delta \subset \mathbb{C}$ be the unit disc. Let $g$ be a univalent holomorphic function on $\Delta$ such that $g(0)=0$ and $g^{\prime}(0)=1$. Then

$$
\Delta_{\frac{1}{4}}:=\left\{z \in \mathbb{C} ;|z|<\frac{1}{4}\right\} \subset g(\Delta) .
$$

With this lemma, we now prove the following:

Theorem 4.2. Let $D$ be a bounded domain in $\mathbb{C}, x \in D$. Then the Carathéodory pseudonorm of $\partial / \partial z$ at $x$ is not less than $s_{D}(x) / 4 \delta(x)$, where $z$ is the standard coordinate on $\mathbb{C}$ and $\partial / \partial z$ is viewed as a vector in the tangent space $T_{x} D$ of $D$ at $x$.

Proof. By Theorem 2.1, there exists a univalent map $f: D \rightarrow \Delta$ such that $f(x)=0$ and $\Delta_{s_{D}(x)} \subset f(D)$, where $\Delta_{S_{D}(x)}$ is the disc in $\mathbb{C}$ with center 0 and radius $s_{D}(x)$. We want to estimate the module $\left|f^{\prime}(x)\right|$ of the derivative of $f$ at $x$. Let

$$
g=\left.f^{-1}\right|_{\Delta_{s_{D}(x)}} .
$$

This is a univalent map from $\Delta_{S_{D}(x)}$ to $D$ such that $g(0)=x$.

Now we define a univalent map $\varphi: \Delta \rightarrow \mathbb{C}$ by setting

$$
\varphi(z)=\frac{g\left(s_{D}(x) \cdot z\right)-x}{s_{D}(x) \cdot g^{\prime}(0)} .
$$

Then it is clear that $\varphi(0)=0$ and $\varphi^{\prime}(0)=1$. By Lemma 4.1, we have $\Delta_{1 / 4} \subset \varphi(\Delta)$. This implies that

$$
\Delta\left(x, \frac{s_{D}(x)\left|g^{\prime}(0)\right|}{4}\right) \subset g\left(\Delta_{s_{D}(x)}\right) \subset D,
$$

where, for $a \in \mathbb{C}$ and $r>0$, we set $\Delta(a, r)$ the disc in $\mathbb{C}$ with center $a$ and radius $r$. In particular, we have

$$
\delta(x) \geq \frac{s_{D}(x)\left|g^{\prime}(0)\right|}{4} .
$$

Noting that $f^{\prime}(x)=1 / g^{\prime}(0)$, we get

$$
\left|f^{\prime}(x)\right| \geq \frac{s_{D}(x)}{4 \delta(x)} .
$$

This means that the Carathéodory pseudonorm of $\partial / \partial z$ at $x$ is not less than

$$
\frac{s_{D}(x)}{4 \delta(x)} \text {. }
$$


Remark 4.3. Using a similar argument as in the proof of Theorem 4.2, one can prove a weaker form of Theorem 4.2 in higher dimensional cases. In fact, for a bounded domain $D \subset \mathbb{C}^{n}$, one can prove that the Carathéodory pseudonorm $\|X\|_{C_{D}}$ on $D$ of $X \in T_{x} D=\mathbb{C}^{n}$ admits the estimate

$$
\|X\|_{C_{D}} \geq \frac{s_{D}(x)\|X\|}{4 \delta(x, X)},
$$

where $\delta(x, X)$ is the boundary distance of $x$ with respect to the direction $X$, and $\|X\|$ is the Euclidean norm of $X$.

A corollary of Theorem 4.2 is the following:

Theorem 4.4. Let $D$ be a bounded domain in $\mathbb{C}$ satisfying

$$
s_{D}(x)>\frac{C}{\log (1 / \delta(x))}
$$

for some positive constant $C$ and all $x \in D$ with $\delta(x)<1$. Then the Carathéodory metric on $D$ is complete.

Proof. By Theorem 4.2, we see that the Carathéodory pseudonorm of $\partial / \partial z$ at $x$ is not less than $-C /(\delta(x) \log \delta(x))$, which implies the completeness of the Carathéodory metric on $D$.

We focus on holomorphic homogeneous regular domains in the rest of this section. We first prove the completeness of the Carathéodory metric of a holomorphic homogeneous regular domain.

Theorem 4.5. Let $D$ be a holomorphic homogeneous regular domain in $\mathbb{C}^{n}$. Then the Carathéodory metric on $D$ is complete.

Proof. Since $D$ is a bounded domain, the Carathéodory metric on $D$ is nondegenerate. Denote by $C_{D}(\cdot, \cdot)$ the Carathéodory distance on $D$.

Let $c>0$ be a positive lower bound of the squeezing function $s_{D}$ of $D$. We first prove that, for any $x_{0} \in D$, the set

$$
A\left(x_{0}\right):=\left\{x \in D \mid C_{D}\left(x, x_{0}\right)<\log \frac{1+c / 2}{1-c / 2}\right\}
$$

is relatively compact in $D$. By Theorem 2.1 , there exists an open holomorphic embedding $f_{x_{0}}: D \rightarrow B^{n}$ such that $f_{x_{0}}\left(x_{0}\right)=0$ and $B^{n}(0, c) \subset f_{x_{0}}(D)$. By the decreasing property of Carathéodory metrics, we have

$$
f_{x_{0}}^{*} C_{B^{n}} \leq C_{D} .
$$

This implies that

$$
f_{x_{0}}\left(A\left(x_{0}\right)\right) \subset\left\{z \in B^{n} \mid C_{B^{n}}(z, 0)<\log \frac{1+c / 2}{1-c / 2}\right\} .
$$


Note that the set

$$
\left\{z \in B^{n} \mid C_{B^{n}}(z, 0)<\log \frac{1+c / 2}{1-c / 2}\right\}=\left\{z \in B^{n} \mid\|z\|<\frac{c}{2}\right\}
$$

is relatively compact in $B^{n}(0, c)$, so $A\left(x_{0}\right)$ is relatively compact in $D$.

For bounded domains, it is known that the topology induced by the Carathéodory metric coincides with the ordinary topology [Jarnicki and Pflug 1993]. In particular, a compact set of $D$ with the ordinary topology is also compact with respect to the topology induced by the Carathéodory metric. By the above result, we see that the Carathéodory metric on $D$ is complete.

It is known that a domain whose Carathéodory metric is complete must be pseudoconvex [Jarnicki and Pflug 1993]; therefore Theorem 4.5, yields the following result, which was proved [Yeung 2009] by a different method:

Corollary 4.6. A holomorphic homogeneous regular domain must be pseudoconvex.

For any complex manifold, it is well known that its Carathéodory pseudometric is always dominated by its Kobayashi pseudometric [Kobayashi 1998]. For bounded domains, a famous result of Look [1958] says that the Carathéodory metric is always dominated by the Bergman metric. Note also that, for a bounded domain $D$, any one of the three intrinsic metrics - the Carathéodory metric, the Kobayashi metric, and the Bergman metric — induces the same topology as the ordinary one. Therefore, as a consequence of Theorem 4.5, we have the following.

Theorem 4.7. Let $D$ be a holomorphic homogeneous regular domain. Then the Kobayashi metric and the Bergman metric on it are complete.

\section{Squeezing functions on planar domains}

In this section, we will consider squeezing functions on planar domains. For finitely connected planar domains, we get a neat description of the boundary behavior of their squeezing functions. As a result, we get the necessary and sufficient condition for such a domain to be a holomorphic homogeneous regular domain. If $D$ has smooth boundary,

$$
\lim _{z \rightarrow \partial D} s_{D}(z)=1 \text {. }
$$

By continuity of $s_{D}$, this implies $D$ is a holomorphic homogeneous regular domain. As a consequence, we can recover some important results about planar domains. For example, the three intrinsic metrics - the Carathéodory metric, the Kobayashi metric, and the Bergman metric — on a bounded planar domain with smooth boundary are all complete, and they are equivalent; all smoothly bounded planar domains are hyperconvex, that is, they admit bounded exhaustive subharmonic functions. 
We also give a class of holomorphic homogeneous regular domains which are infinitely connected.

It is clear that the squeezing function of the unit disc is the constant function with value 1. By the Riemann mapping theorem and holomorphic invariance of squeezing functions, the squeezing function of any simply connected bounded planar domain is also constant with value 1 .

Now we consider squeezing functions on 2-connected planar domains. Define

$$
A_{r}=\{z \in \mathbb{C}|r<| z \mid<1\}
$$

for $0 \leq r<1$. When $r>0$, we call $A_{r}$ an annulus. It is well-known that a 2-connected domain in $\mathbb{C}$ which is not conformal equivalent to $\mathbb{C}^{*}$ must be holomorphic equivalent to a unique $A_{r}$ [Ahlfors 1978]. When $r=0, A_{0}$ is just the punctured disc $\Delta^{*}$, a case we will consider it in the last section. For $A_{r}$ with $r>0$, we have the following.

Theorem 5.1. For $r>0$, the squeezing function $s_{A_{r}}(z)$ tends to 1 as $z \rightarrow \partial A_{r}$. In particular, $A_{r}$ is a holomorphic homogeneous regular domain.

Proof. For $c \in[0,1)$, we define

$$
\sigma(c)=\log \frac{1+c}{1-c} .
$$

It is clear that $\sigma(c)$ is strictly increasing for $0 \leq c<1$, and its inverse is given by $\sigma^{-1}(w)=\tanh (w / 2)$. For a point $z \in \Delta$, the Poincaré distance from 0 to $z$ is $\sigma(|z|)$.

Now let $z \in A_{r}$. With respect to the Poincare metric on $\Delta$, the distance from $z$ to the cycle $\{w ;|w|=r\}$ is $\sigma(|z|)-\sigma(r)$. Denote by $P(z, \sigma(|z|)-\sigma(r))$ the disc (with respect to the Poincaré metric on $\Delta$ ) with center $z$ and radius $\sigma(|z|)-\sigma(r)$. Then we have $P(z, \sigma(|z|)-\sigma(r)) \subset A_{r}$. Choose a conformal map $f: \Delta \rightarrow \Delta$ such that $f(z)=0$. Since $f$ preserves the Poincaré metric on $\Delta$, it maps $P(z, \sigma(|z|)-\sigma(r))$ onto the disc (with respect to the Poincaré metric on $\Delta$ ) with center $f(z)=0$ and radius $\sigma(|z|)-\sigma(r)$, which is just the Euclidean disc with center 0 and radius $\sigma^{-1}(\sigma(|z|)-\sigma(r))$. This implies that

$$
s_{A_{r}}(z) \geq \sigma^{-1}(\sigma(|z|)-\sigma(r)) .
$$

Note that $\sigma^{-1}(\sigma(|z|)-\sigma(r)) \rightarrow 1$ as $|z| \rightarrow 1$, so $s_{A_{r}}(z)$ tends to 1 as $|z| \rightarrow$ 1. Consider the holomorphic automorphism of $A_{r}$ given by $z \mapsto r / z$. By the conformal invariance of $s_{A_{r}}$, we also get $s_{A_{r}}(z)$ tends to 1 as $|z| \rightarrow r$.

By Theorem 3.1, $s_{A_{r}}$ is continuous and hence has a positive lower bound, so $A_{r}$ is a holomorphic homogeneous regular domain.

By a similar argument, Theorem 5.1 can be generalized to finitely connected planar domains as follows: 
Theorem 5.2. Let $D$ be a domain in $\mathbb{C}$. Assume that $\overline{\mathbb{C}}-D$ has finitely many connected components such that each connected component is not a single point. Then we have

$$
\lim _{z \rightarrow \partial D} s_{D}(z)=1 .
$$

In particular, $D$ is a holomorphic homogeneous regular domain.

Proof. We define the function $\sigma$ as in the proof of Theorem 5.1. Let $E_{1}, \ldots, E_{n}$ be connected components of $\overline{\mathbb{C}}-D$. Then $D_{1}:=\overline{\mathbb{C}}-E_{1}$ is simply connected and $D \subset D_{1}$. Since $E_{1}$ is not a single point, by Riemann mapping theorem, there is a conformal map $\varphi_{1}$ from $D_{1}$ to $\Delta$. It is clear that $\varphi_{1}(D)$ is a domain in $\Delta$ obtained by deleting $n-1$ connected compact subsets, say $L_{2}, \ldots, L_{n}$, from $\Delta$.

Let $z \in D$ and let $l_{z}=P_{\Delta}\left(z, \cup_{i=2}^{n} L_{i}\right)$ be the distance from $z$ to $\cup_{i=2}^{n} L_{i}$ with respect to the Poincaré distance of $\Delta$. Choose a conformal map $f: \Delta \rightarrow \Delta$ such that $f(z)=0$. Then the Euclidean disc with center 0 and radius $\sigma^{-1}\left(l_{z}\right)$ is contained in $f(\varphi(D))$, which implies that $s_{\varphi(D)}(z) \geq \sigma^{-1}\left(l_{z}\right)$. If $|z|$ tends to 1 , then $l_{z}$ tends to $\infty$ and $\sigma^{-1}\left(l_{z}\right)$ tends to 1 . Hence $s_{\varphi(D)}(z)$ tends to 1 . By holomorphic invariance of squeezing functions, we see that

$$
\lim _{z \rightarrow E_{1}} s_{D}(z)=1 .
$$

Similarly, for $E_{i}$ with $2 \leq i \leq n$, we have

$$
\lim _{z \rightarrow E_{i}} s_{D}(z)=1 .
$$

Hence

$$
\lim _{z \rightarrow \partial D} s_{D}(z)=1 .
$$

By continuity of $s_{D}$, a positive lower bound is admitted on $D$, so $D$ is a holomorphic homogeneous regular domain.

Using the Riemann mapping theorem, one can prove that the domains considered in the above theorem are holomorphic equivalent to bounded domains with smooth boundary [Ahlfors 1978]. Hence an equivalent version of the above theorem is the following:

Theorem 5.3. Let $D$ be a bounded domain in $\mathbb{C}$ with smooth boundary. We have

$$
\lim _{z \rightarrow \partial D} s_{D}(z)=1
$$

In particular, $D$ is a holomorphic homogeneous regular domain.

Remark 5.4. As a consequence of Theorem 5.3, for a bounded planar domain $D$ with smooth boundary, $s_{D}$ can be extended continuously to $\bar{D}$. It may be interesting to investigate whether the same result holds in higher dimensions. 
In Section 3, we have shown that the Carathéodory metric, the Kobayashi metric, and the Bergman metric on a holomorphic homogeneous regular domain are complete. As mentioned in the introduction, these intrinsic metrics on a holomorphic homogeneous regular domain are equivalent [Liu et al. 2004; Liu et al. 2005]. It also turns out that any holomorphic homogeneous regular domain is hyperconvex [Yeung 2009], so, as a result of Theorem 5.3, we can recover the following results in complex analysis:

Theorem 5.5. Let $D$ be a planar domain with smooth boundary. Then

(1) the Carathéodory metric, the Kobayashi metric and the Bergman metric on D are complete;

(2) The Carathéodory metric, the Kobayashi metric and the Bergman metric on $D$ are equivalent;

(3) D is hyperconvex.

By definition, it is clear that the product of two holomorphic homogeneous regular domains is again a holomorphic homogeneous regular domain. So we get the following.

Corollary 5.6. Let $D$ be a bounded domain in $\mathbb{C}^{n}$ which is holomorphic equivalent to the product of bounded planar domains with smooth boundary. Then $D$ is a holomorphic homogeneous regular domain.

As mentioned in the introduction, the list of known holomorphic homogeneous regular domains contains bounded homogeneous domains, Teichmüller spaces, bounded domains covering compact Kähler manifolds, and strictly convex domains with $C^{2}$-boundary. Many examples of holomorphic homogeneous regular domains given by Corollary 5.6 are not in the list. More precisely, we have the following.

Proposition 5.7. Let $D_{1}, \ldots, D_{k}$ be bounded planar domains with smooth boundaries which are mutually not conformal equivalent. If there exists a $D_{i}$ which is not conformal equivalent to the unit disc $\Delta$, the domain $D:=D_{1}^{r_{1}} \times \cdots \times D_{k}^{r_{k}}$ is a holomorphic homogeneous regular domain which is not holomorphic equivalent to any of the domains in the above list, where $r_{1}, \ldots, r_{k}$ are positive integers and $D_{i}^{r_{i}}=D_{i} \times \cdots \times D_{i}$ is the $r_{i}$-power of $D_{i}$.

Proof. Denote by $\operatorname{Aut}(D)$ the holomorphic automorphism group of $D$. By the proposition in [Royden 1974] and [Urata 1981, Theorem 1], we have

$$
\operatorname{Aut}(D)=\operatorname{Aut}\left(D_{1}^{r_{1}}\right) \times \cdots \times \operatorname{Aut}\left(D_{k}^{r_{k}}\right),
$$

and, for each $i$, the following sequence is exact:

$$
1 \rightarrow\left(\operatorname{Aut}\left(D_{i}\right)\right)^{r_{i}} \rightarrow \operatorname{Aut}\left(D_{i}^{r_{i}}\right) \rightarrow S_{r_{i}} \rightarrow 1,
$$


where $S_{r_{i}}$ is the symmetry group of degree $r_{i}$ which acts on $D_{i}^{r_{i}}$ by permutation. The decomposition of $\operatorname{Aut}(D)$ implies that $D$ is homogeneous if and only if each factor $D_{i}$ is homogeneous, and $D$ can cover a compact complex manifold if and only if each $D_{i}$ does so too. Note that a smoothly bounded planar domain is homogeneous or can cover a compact complex manifold if and only if it is isomorphic to $\Delta$. Hence $D$ can not be homogeneous or cover a compact complex manifold.

It is clear that $D$ can not be holomorphic equivalent to any convex domain since the fundamental group of $D$ is nontrivial. By the same reasoning, $D$ is not holomorphic equivalent to any Teichmüller space since it is well known that all Teichmüller spaces are contractible.

For general finitely connected planar domains, the boundary behavior of their squeezing functions can be described as follows:

Theorem 5.8. Let $D$ be a finitely connected planar domain, and $E$ a connected component of $\overline{\mathbb{C}}-D$.

(1) if $E$ is not a single point,

$$
\lim _{D \ni z \rightarrow E} s_{D}(z)=1 .
$$

(2) if $E=\{p\}$ contains a single point,

$$
s_{D}(z) \leq \sigma^{-1}\left(K_{\widetilde{D}}(z, p)\right), \quad z \in D,
$$

where $\sigma$ is defined as in the proof of Theorem 5.1 and $K_{\widetilde{D}}(\cdot, \cdot)$ is the Kobayashi distance on the domain $\widetilde{D}:=D \cup\{p\}$. In particular,

$$
\lim _{D \ni z \rightarrow p} s_{D}(z)=0 .
$$

Proof. The proof of (1) is similar to the proof of Theorem 5.2 and we will not repeat it here. Now we give the proof of (2). Let $z \in D$, and $f_{z}: D \rightarrow \Delta$ be a holomorphic embedding such that $f_{z}(z)=0$. Note that $\widetilde{D}$ is a domain. By Riemann's removable singularity theorem, $f_{z}$ can be extended as a holomorphic map $\tilde{f}_{z}$ from $\widetilde{D}$ to $\Delta$. It is clear that $\tilde{f}_{z}(p) \notin f(D)$. By the decreasing property of Kobayashi distances, we have $K_{\Delta}\left(0, \tilde{f}_{z}(p)\right) \leq K_{\widetilde{D}}(z, p)$. Hence $s_{D}(z) \leq \sigma^{-1}\left(K_{\widetilde{D}}(z, p)\right)$.

Remark 5.9. Theorem 5.8 implies that a finitely connected planar domain is a holomorphic homogeneous regular domain if and only if each connected component of its complement in $\overline{\mathbb{C}}$ is not a single point.

The examples of planar holomorphic homogeneous regular domains given by Theorem 5.2 are all finitely connected, that is, their complement in $\overline{\mathbb{C}}$ have finitely many connected components. We can also construct a class of planar holomorphic homogeneous regular domains which are infinitely connected. 
Let $\operatorname{Aut}(\Delta)$ be the holomorphic automorphism group of $\Delta$, and denote by $\Delta_{r}$ the disc in $\mathbb{C}$ with center 0 , radius $r$, and $\bar{\Delta}_{r}$ its closure. We first prove the following.

Lemma 5.10. For any positive numbers $u$, $v$, and $w$ with $u<v<w<1$, there exists a positive number $c(u, v, w)$ such that for an arbitrary positive number $r$ with $u<r<v$, letting $D \subset A_{r}$ be a domain containing $\Delta_{w} \backslash \bar{\Delta}_{r}$, we have $s_{D}(z) \geq$ $c(u, v, w)$ for $z \in \Delta_{v} \backslash \bar{\Delta}_{r}$.

Proof. Consider the reflection $R: D \rightarrow \Delta$ given by

$$
z \mapsto \frac{r}{z}
$$

It is clear that $z \in R(D)$, provided $r / w<|z|<1$. For $z \in \Delta_{v} \backslash \bar{\Delta}_{r}$, we have

$$
r / v<|R(z)|<1 \text {. }
$$

Hence the disc (with respect to the Poincaré metric) with center $z$ and radius $\sigma(r / v)-\sigma(r / w)$ is contained in $R(D)$. So we see that

$$
s_{R(D)}(R(z))<\sigma^{-1}\left(\sigma\left(\frac{r}{v}\right)-\sigma\left(\frac{r}{w}\right)\right)
$$

for $z \in \Delta_{v} \backslash \bar{\Delta}_{r}$. By the biholomorphic invariance of squeezing functions, we get

$$
s_{D}(z) \geq \sigma^{-1}\left(\sigma\left(\frac{r}{v}\right)-\sigma\left(\frac{r}{w}\right)\right)
$$

for $z \in \Delta_{v} \backslash \bar{\Delta}_{r}$. Take

$$
c(u, v, w)=\inf _{u \leq r \leq v}\left\{\sigma^{-1}\left(\sigma\left(\frac{r}{v}\right)-\sigma\left(\frac{r}{w}\right)\right)\right\} .
$$

it is clear that $c(u, v, w)>0$ and it satisfies the condition of the lemma.

By the above lemma, we can prove the following

Theorem 5.11. Let $u, v$, and $w$ be positive numbers with $u<v<w<1$. Let $r_{k}$, $k=1,2, \ldots$, be a sequence of positive numbers satisfying $u<r_{k}<v$. Let $f_{k}$ be a sequence in $\operatorname{Aut}(\Delta)$ such that the $f_{k}\left(\bar{\Delta}_{w}\right)$ are pairwise disjoint. Then the domain

$$
D=\Delta \backslash\left(\cup_{k=1}^{\infty} f_{k}\left(\bar{\Delta}_{r_{k}}\right)\right)
$$

is a holomorphic homogeneous regular domain.

Proof. Let $c(u, v, w)$ be the same as in Lemma 5.10. Denote $c(u,(v+w) / 2, w)$ by $c$. By the above lemma and biholomorphic invariance of squeezing functions, we have $s_{D}(z) \geq c$ for

$$
z \in D^{\prime}:=D \cap\left(\cup_{k=1}^{\infty} f_{k}\left(\Delta_{(v+w) / 2}\right)\right) .
$$


For $z \in D \backslash D^{\prime}$, the distance from $z$ to $\partial D$ with respect to the Poincaré distance on $\Delta$ is greater than $\sigma((v+w) / 2)-\sigma(v)$. Taking a conformal map $f \in \operatorname{Aut}(\Delta)$ such that $f(z)=0$, we see that

$$
s_{D}(z) \geq \sigma^{-1}\left(\sigma\left(\frac{v+w}{2}\right)-\sigma(v)\right) .
$$

So $s_{D}$ has a positive lower bound, and hence $D$ is a holomorphic homogeneous regular domain.

As a consequence, the Carathéodory metrics, the Kobayashi metrics, and the Bergman metrics on domains that are constructed in Theorem 5.11 are complete and equivalent. An explicit example can be constructed as follows: let $f \in \operatorname{Aut}(\Delta)$ be defined by

$$
f(z)=\frac{z+1 / 2}{1+z / 2}
$$

and let $D=\Delta \backslash\left(\cup_{k=-\infty}^{\infty} f^{k}\left(\bar{\Delta}_{1 / 4}\right)\right)$. Then $D$ is a holomorphic homogeneous regular domain, and the Carathéodory metric, the Kobayashi metric, and the Bergman metric on $D$ are complete and equivalent. The special domain $D$ was constructed in [Krantz 1990] to show that a bounded planar domain may have an infinite discrete automorphism group.

\section{Squeezing functions on annuli}

In the above section, we have studied some properties of squeezing functions of annuli. In this section, we want to further investigate their properties.

We have seen that all annuli are holomorphic homogeneous regular domains, and their squeezing functions tend to 1 at the boundary. An interesting but difficult problem is to give an exact expression of $s_{A_{r}}$. Another relatively simple problem is to determine the minimum of $s_{A_{r}}$ for $r>0$, which are conformal invariants.

By the conformal invariance of $s_{A_{r}}, s_{A_{r}}(z)$ depends only on $|z|$, so $s_{A_{r}}$ is reduced to a function defined on $(r, 1)$. Be the reflection $z \mapsto r / z$, it can be further reduced to a function on $[\sqrt{r}, 1)$. We show that $s_{A_{r}}(x)$ is strictly increasing on $[\sqrt{r}, 1)$. To prove this result, we first prove two propositions, which are also interesting in their own right.

Let $D \subset \mathbb{C}$ be a bounded domain with smooth boundary, denote by $H_{D}$ the set of univalent maps $f$ from $D$ to $\Delta$ such that $\Delta \backslash f(D)$ is a compact set. For bounded planar domain $D$, we always denote by $P_{D}(\cdot, \cdot)$ the Poincaré distance of $D$.

Proposition 6.1. Let $D \subset \mathbb{C}$ be a bounded domain with smooth boundary. Then, for any $p \in D$, we have

$$
s_{D}(p)=\sup \left\{r \mid \Delta_{r} \subset f(D) \text { for some } f \in H_{D} \text { with } f(p)=0\right\} .
$$


Proof. Let $f: D \rightarrow \Delta$ be a univalent map with $f(p)=0$. Assume $\Delta_{r} \subset f(D)$. Let $D^{\prime}$ be the union of $f(D)$ and the compact connected components of $\Delta \backslash f(D)$. Then $D^{\prime}$ is simply connected. By the Riemann mapping theorem, there is a conformal map $g: D^{\prime} \rightarrow \Delta$ with $g(0)=0$.

Note that

$$
\Delta_{r}=\left\{z \in \Delta \mid P_{\Delta}(z, 0)<\sigma(r)\right\} .
$$

By the decreasing property of the Poincare metrics on planar domains, we have $P_{\Delta}(z, w) \leq P_{D^{\prime}}(z, w)$ for all $z, w \in D^{\prime}$, so

$$
\left\{z \in D^{\prime} \mid P_{D^{\prime}}(z, 0)<\sigma(r)\right\} \subset \Delta_{r} .
$$

Note that $g$ is an isometry from $\left(D^{\prime}, P_{D^{\prime}}\right)$ to $\left(\Delta, P_{\Delta}\right)$. Hence

$$
\Delta_{r}=\left\{z \in \Delta \mid P_{\Delta}(z, 0)<\sigma(r)\right\}=g\left(\left\{z \in D^{\prime} \mid P_{D^{\prime}}(z, 0)<\sigma(r)\right\}\right) \subset g(f(D)) .
$$

Now we get a univalent map $g \circ f: D \rightarrow \Delta$ with $g \circ f \in H_{D}, g \circ f(p)=0$, and $\Delta_{r} \subset g \circ f(D)$. Since $f$ is arbitrary, we see that

$$
s_{D}(p)=\sup \left\{r \mid \Delta_{r} \subset h(D) \text { for some } h \in H_{D} \text { with } h(p)=0\right\} .
$$

For two subsets $A$ and $B$ of $\Delta$, we denote by $P_{\Delta}(A, B)$ the distance between $A$ and $B$ with respect to the Poincaré distance of $\Delta$.

Proposition 6.2. Let $D \subset \mathbb{C}$ be a bounded domain with smooth boundary. For $p \in D$, let

$$
u(p)=\sup \left\{P_{\Delta}(f(p), \Delta \backslash f(D)) \mid f \in H_{D}\right\} .
$$

Then we have $s_{D}(p)=\sigma^{-1}(u(p))$.

Proof. For $p \in D$ and $f \in H_{D}$, let

$$
u=P_{\Delta}(f(p), \Delta \backslash f(D)) .
$$

Then the $P_{\Delta}$-disc with center $f(p)$ and radius $u$ is contained in $f(D)$. Take a conformal map $g: \Delta \rightarrow \Delta$ such that $g(f(p))=0$. Then $\Delta_{\sigma^{-1}(u)} \subset g \circ f(D)$, so we have $s_{D}(p) \geq \sigma^{-1}(u)$. Since $f \in H_{D}$ is arbitrary, we have $s_{D}(p) \geq \sigma^{-1}(u(p))$.

On the other hand, for an arbitrary $f \in H_{D}$ with $f(p)=0$, let $r$ be the positive number such that $\Delta_{r} \in f(D)$ but $\Delta_{r+\epsilon} \nsubseteq f(D)$ for any $\epsilon>0$. It is clear that

$$
r=\sigma^{-1}\left(P_{\Delta}(f(p), \Delta \backslash f(D))\right) \leq \sigma^{-1}(u(p)) .
$$

Since $f \in H_{D}$ is arbitrary, by the above proposition, we have

$$
s_{D}(p) \leq \sigma^{-1}(u(p)) .
$$

Theorem 6.3. Viewed as a function on $[\sqrt{r}, 1)$, the squeezing function $s_{A_{r}}(z)$ of $A_{r}$ is strictly increasing on $[\sqrt{r}, 1)$; in particular, it attains its minimum at $\sqrt{r}$. 
Proof. For simplicity, let $s=s_{A_{r}}$. For $x \in[\sqrt{r}, 1)$, by Theorem 2.1, there is a univalent map $f: D \rightarrow \Delta$ such that $f(x)=0$ and $\Delta_{s(x)} \subset f(D)$. By Proposition 6.1, we may assume $f \in H_{D}$. By Proposition 6.2, we have

$$
s(x)=\sigma^{-1}\left(P_{\Delta}(f(x), \Delta \backslash f(D))\right) .
$$

By Proposition 6.2 and the conformal invariance of $s$, we have the identity

$$
P_{\Delta}(f(x), \Delta \backslash f(D))=\sup \left\{P_{\Delta}(f(z), \Delta \backslash f(D))|z \in D,| z \mid=x\right\} .
$$

Note that the curve $f(|z|=\sqrt{r})$ separates $\mathbb{C}$ into two connected parts. Let $U$ and $V$ be the bounded and unbounded connected components of $\mathbb{C} \backslash f(|z|=\sqrt{r})$, respectively.

If $x>\sqrt{r}$, then $f(|z|=x) \subset V$. In fact, if it is not the case, then composing $f$ with the reflection $z \mapsto r / z$ will lead to a contradiction to the extremal property assumption on $f$.

Now let $x^{\prime} \in[\sqrt{r}, 1)$ with $x^{\prime}>x$. Then it is clear that $f\left(|z|=x^{\prime}\right)$ lies in the unbounded component of $\mathbb{C} \backslash f(|z|=x)$, so we have

$\sup \left\{P_{\Delta}(f(z), \Delta \backslash f(D))|z \in D| z \mid,=x^{\prime}\right\}>\sup \left\{P_{\Delta}(f(z), \Delta \backslash f(D))|z \in D| z \mid,=x\right\}$.

By Proposition 6.2, there is a point $z \in A_{r}$ with $|z|=x^{\prime}$ and $s_{A_{r}}(z)>s(x)$. Note that $s_{A_{r}}(z)=s(|z|)=s\left(x^{\prime}\right)$. Hence $s\left(x^{\prime}\right)>s(x)$.

Theorem 6.3 and its proof lead us to conjecture that, for $\rho \in[\sqrt{r}, 1), s_{A_{r}}(\rho)$ is given by

$$
s_{A_{r}}(\rho)=\sigma^{-1}(\sigma(\rho)-\sigma(r))=\sigma^{-1}\left(\log \frac{(1+\rho)(1-r)}{(1-\rho)(1+r)}\right),
$$

where the function $\sigma$ is defined as in the proof of Theorem 5.1. Provided this conjecture, Theorem 6.3 implies that $s_{A_{r}}(\rho)$ attains its minimum

$$
s_{A_{r}}(\sqrt{r})=\tanh \log \frac{1+\sqrt{r}}{\sqrt{1+r}}
$$

at $\rho=\sqrt{r}$, which characterizes the conformal structure of $A_{r}$.

\section{Explicit form of squeezing functions on some special domains}

In this section, we give the explicit form of squeezing functions on some special domains. Namely, punctured balls and classical bounded symmetric domains.

We first consider domains constructed by deleting analytic subsets from other domains. 
Theorem 7.1. Let $D^{\prime} \subset \mathbb{C}^{n}$ be a bounded domain and $A \subset D^{\prime}$ be a proper analytic subset. Then, for the domain $D=D^{\prime} \backslash A$, we have

$$
s_{D}(z) \leq \sigma^{-1}\left(K_{D^{\prime}}(z, A)\right), \quad z \in D,
$$

where $\sigma$ is defined as in the proof of Theorem 5.1 and $K_{D^{\prime}}(\cdot, \cdot)$ is the Kobayashi distance on $D^{\prime}$; in particular, we have $\lim _{z \rightarrow A} s_{D}(z)=0$.

Proof. Let $z \in D$, and $f_{z}: D \rightarrow B^{n}$ be a holomorphic open embedding such that $f_{z}(z)=0$. By Riemann's removable singularity theorem, $f_{z}$ can be extended to a holomorphic map $\tilde{f}_{z}$ from $D^{\prime}$ to $B^{n}$. It is clear that $\tilde{f}_{z}(D) \cap \tilde{f}_{z}(A)=\varnothing$. By the decreasing property of the Kobayashi distance, we have $K_{B^{n}}\left(0, \tilde{f}_{z}(A)\right) \leq$ $K_{D^{\prime}}(z, A)$. Hence $s_{D}(z) \leq \sigma^{-1}\left(K_{D^{\prime}}(z, A)\right), z \in D$.

Remark 7.2. The domains $D$ constructed in the above theorem are not holomorphic homogeneous regular domains. The conclusion can also be derived from Corollary 4.6, since $D$ is not hyperconvex, or Theorem 4.5, since the Carathéodory metric on $D$, which is just the restriction of the Carathéodory metric of $D^{\prime}$, is not complete.

For the special case of punctured balls, Theorem 7.1 implies the following.

Corollary 7.3. The squeezing function $s_{B^{n}} \backslash\{0\}$ on the $n$ dimensional punctured ball $B^{n} \backslash\{0\}$ is given by

$$
s_{B^{n} \backslash\{0\}}(z)=\|z\|,
$$

where $\|z\|$ is the Euclidean norm of $z$.

Proof. By Theorem 7.1, we have $s_{B^{n} \backslash\{0\}}(z) \leq\|z\|$. On the other hand, it is clear that $s_{B^{n} \backslash\{0\}}(z) \geq\|z\|$. Hence $s_{B^{n} \backslash\{0\}}(z)=\|z\|$.

Other examples of bounded domains whose squeezing functions can be given explicitly are classical symmetric bounded domains. Recall that a classical symmetric domain is a domain of one of the following four types:

$D_{\mathrm{I}}(r, s)=\left\{Z=\left(z_{j k}\right): I-Z \bar{Z}^{\prime}>0\right.$, where $Z$ is an $r \times s$ matrix $\}(r \leq s)$,

$D_{\mathrm{II}}(p)=\left\{Z=\left(z_{j k}\right): I-Z \bar{Z}^{\prime}>0\right.$, where $Z$ is a symmetric matrix of order $\left.p\right\}$,

$D_{\mathrm{III}}(q)=\left\{Z=\left(z_{j k}\right): I-Z \bar{Z}^{\prime}>0\right.$, where $Z$ is a skew-symmetric matrix of order $\left.q\right\}$, $D_{\mathrm{IV}}(n)=\left\{z=\left(z_{1}, \ldots, z_{n}\right) \in \mathbb{C}^{n}: 1+\left|z z^{\prime}\right|^{2}-2 z z^{\prime}>0,1-\left|z z^{\prime}\right|>0\right\}$.

Here $I$ is the identity matrix of proper order, $\bar{Z}$ denotes the conjugate matrix of $Z$, and $Z^{\prime}$ denotes the transposed matrix of $Z$. The complex dimensions of these four domains are $r s, p(p+1) / 2, q(q-1) / 2$, and $n$, respectively.

Given a bounded homogeneous domain $D$, by the holomorphic invariance of squeezing functions, $s_{D}$ is a constant function on $D$, and we denote this constant by 
$s(D)$. By a theorem of Kubota based on [Alexander 1978], the squeezing functions on the above four types of domains can be given explicitly as follows:

Theorem 7.4 [Kubota 1982c, Theorem 1].

$$
\begin{aligned}
& s\left(D_{I}(r, s)\right)=r^{-\frac{1}{2},} \\
& s\left(D_{I I}(p)\right)=p^{-\frac{1}{2}}, \\
& s\left(D_{I I I}(q)\right)=\left[\frac{q}{2}\right]^{-\frac{1}{2}}, \\
& s\left(D_{I V}(n)\right)=2^{-\frac{1}{2},}
\end{aligned}
$$

where $[q / 2]$ denotes the integral part of $q / 2$.

For products of classical symmetric domains, we have the following:

Theorem 7.5 [Kubota 1982c, Theorem 2]. If $D_{1}, \ldots, D_{m}$ are classical symmetric domains, then

$$
s\left(D_{1} \times \cdots \times D_{m}\right)=\left(s\left(D_{1}\right)^{-2}+\cdots+s\left(D_{m}\right)^{-2}\right)^{-\frac{1}{2}} .
$$

Remark 7.6. Kubota [1981; 1982a; 1982b; 1982c; 1983] considered the following Carathéodory extremal problem:

$$
M\left(z_{0}, D\right)=\sup _{F \in \mathfrak{F}(D)}\left|J_{F}\left(z_{0}\right)\right|, \quad\left(z_{0} \in D\right),
$$

where $D$ is a bounded domain in the complex Euclidean space $\mathbb{C}^{n}$ and $\mathfrak{F}(D)$ consists of all holomorphic mappings from $D$ into the unit ball $B^{n}$ in $\mathbb{C}^{n}$, and $J_{F}$ is the Jacobian of $F$.

He proved that the extremal mapping of the extremal problem (3) is unique up to a unitary linear transformation when $D$ is a bounded symmetric domain (including two exceptional cases) [Kubota 1983]. We observe that the extremal mappings are exactly the extremal embedding from bounded symmetric domains into the unit ball. Take $D_{I}(r, s)$ here for example, we can find from Kubota's proof that the extremal mapping is $f(z)=z / \sqrt{r}, z=\left(z_{11}, \ldots, z_{1 s}, z_{21}, \ldots, z_{r s}\right) \in \mathbb{C}^{r s}$, which is exactly an extremal embedding for the squeezing function $s\left(D_{I}(r, s)\right)=r^{-1 / 2}$, since one knows $B_{n} \subset D_{I}(r, s) \subset \sqrt{r} B^{n},(n=r s)$.

Ma [1997] considered the extremal problem (3) when $D$ is a complex ellipsoid in $\mathbb{C}^{n}$, that is,

$$
D=D\left(k_{1}, \ldots, k_{n}\right)=\left\{\left.z \in \mathbb{C}^{n}\left|\sum_{j=1}^{n}\right| z_{j}\right|^{k_{j}}<1\right\},
$$

where $k_{j}(j=1,2, \ldots, n)$ are positive real numbers. It is proved that the extremal mapping is again linear, and we conjecture that it is likely the extremal embedding for squeezing function $s_{D}(z)$ in this case. Therefore, it will be interesting to 
consider relations in general between squeezing function $s_{D}$ on a bounded domain $D$ and the Carathéodory maps from $D$ into the unit ball, especially when $D$ is homeomorphic to a cell.

\section{Acknowledgements}

The authors thank Xiangyu Zhou, the PhD advisor of the first two authors, for invaluable instruction and discussions. They are also grateful to Boyong Chen, Kefeng Liu, Peter Pflug, and Sai-Kee Yeung for helpful discussions. The authors thank the referee for many remarkable comments and suggestions.

\section{References}

[Ahlfors 1973] L. V. Ahlfors, Conformal invariants: topics in geometric function theory, McGrawHill, New York, 1973. MR 50 \#10211 Zbl 0272.30012

[Ahlfors 1978] L. V. Ahlfors, Complex analysis: an introduction to the theory of analytic functions of one complex variable, 3rd ed., McGraw-Hill, New York, 1978. MR 80c:30001 Zbl 0395.30001

[Alexander 1978] H. Alexander, "Extremal holomorphic imbeddings between the ball and polydisc", Proc. Amer. Math. Soc. 68:2 (1978), 200-202. MR 57 \#12914 Zbl 0379.32022

[Barth 1972] T. J. Barth, "The Kobayashi distance induces the standard topology", Proc. Amer. Math. Soc. 35:2 (1972), 439-441. MR 46 \#5668 Zbl 0259.32007

[Fisher 1983] S. D. Fisher, Function theory on planar domains: a second course in complex analysis, Wiley, New York, 1983. MR 85d:30001 Zbl 0511.30022

[Fritzsche and Grauert 2002] K. Fritzsche and H. Grauert, From holomorphic functions to complex manifolds, Graduate Texts in Mathematics 213, Springer, New York, 2002. MR 2003g:32001 Zbl 1005.32002

[Hahn 1976] K. T. Hahn, "Quantitative Bloch's theorem for certain classes of holomorphic mappings of the ball into $P_{n}(C)$ ", J. Reine Angew. Math. 283-284 (1976), 99-109. MR 53 \#885 Zbl 0344.32013

[Jarnicki and Pflug 1993] M. Jarnicki and P. Pflug, Invariant distances and metrics in complex analysis, De Gruyter Expositions in Mathematics 9, De Gruyter, Berlin, 1993. MR 94k:32039 Zbl 0789.32001

[Kobayashi 1998] S. Kobayashi, Hyperbolic complex spaces, Grundlehren der Mathematischen Wissenschaften 318, Springer, Berlin, 1998. MR 99m:32026 Zbl 0917.32019

[Krantz 1990] S. G. Krantz, Complex analysis: the geometric viewpoint, Carus Mathematical Monographs 23, Mathematical Association of America, Washington, DC, 1990. MR 92a:30026 Zbl 0743. 30002

[Kubota 1981] Y. Kubota, "An extremal problem on the classical Cartan domains, I", Kodai Math. J. 4:2 (1981), 278-287. MR 83h:32031 Zbl 0486.32021

[Kubota 1982a] Y. Kubota, "An extremal problem on the classical Cartan domains, II", Kodai Math. J. 5:2 (1982), 218-224. MR 84e:32033a Zbl 0502.32023

[Kubota 1982b] Y. Kubota, "An extremal problem on the classical Cartan domains, III", Kodai Math. J. 5:3 (1982), 402-407. MR 84e:32033b Zbl 0515.32016

[Kubota 1982c] Y. Kubota, "A note on holomorphic imbeddings of the classical Cartan domains into the unit ball”, Proc. Amer. Math. Soc. 85:1 (1982), 65-68. MR 83i:32036 Zbl 0459.32015 
[Kubota 1983] Y. Kubota, "An extremal problem on bounded symmetric domains", Bull. London Math. Soc. 15:2 (1983), 126-130. MR 84g:32047 Zbl 0487.32018

[Liu et al. 2004] K. Liu, X. Sun, and S.-T. Yau, "Canonical metrics on the moduli space of Riemann surfaces, I”, J. Differential Geom. 68:3 (2004), 571-637. MR 2007g:32009 Zbl 1078.30038

[Liu et al. 2005] K. Liu, X. Sun, and S.-T. Yau, "Canonical metrics on the moduli space of Riemann surfaces, II”, J. Differential Geom. 69:1 (2005), 163-216. MR 2007g:32010 Zbl 1086.32011

[Lloyd 1979] N. G. Lloyd, "Remarks on generalising Rouché's theorem”, J. London Math. Soc. (2) 20:2 (1979), 259-272. MR 80m:32029 Zbl 0407.32002

[Look 1958] K. H. Look, "Schwarz lemma and analytic invariants", Sci. Sinica 7 (1958), 453-504. MR 21 \#5028 Zbl 0085.06803

[Ma 1997] D. Ma, "Carathéodory extremal maps of ellipsoids", J. Math. Soc. Japan 49:4 (1997), 723-739. MR 98e:32043 Zbl 0917.32017

[Remmert 1998] R. Remmert, Classical topics in complex function theory, Graduate Texts in Mathematics 172, Springer, New York, 1998. MR 98g:30002 Zbl 0895.30001

[Royden 1974] H. L. Royden, "Holomorphic fiber bundles with hyperbolic fiber", Proc. Amer. Math. Soc. 43:2 (1974), 311-312. MR 49 \#3229 Zbl 0284.32017

[Urata 1981] T. Urata, "Holomorphic automorphisms and cancellation theorems", Nagoya Math. J. 81 (1981), 91-103. MR 82h:32029 Zbl 0416.32011

[Yeung 2009] S.-K. Yeung, "Geometry of domains with the uniform squeezing property", Adv. Math. 221:2 (2009), 547-569. MR 2010b:32034 Zbl 1165.32004

Received June 18, 2011. Revised September 28, 2011.

FUSHENG DENG

SCHOOL of Mathematical SCIENCES

GRADUATE UNIVERSITY OF CHINESE ACADEMY OF SCIENCES

BEIJING 100049

CHINA

fshdeng@gucas.ac.cn

QIAN GUAN

BeiJing International Center for Mathematical Research

PEKING UNIVERSITY

BEIJING 100871

CHINA

guanqian@amss.ac.cn

LIYOU ZHANG

SCHOOL OF Mathematical SCIENCES

CAPITAL NORMAL UNIVERSITY

BEIJING 100048

CHINA

zhangly@mail.cnu.edu.cn 


\title{
PACIFIC JOURNAL OF MATHEMATICS
}

\author{
http://pacificmath.org \\ Founded in 1951 by \\ E. F. Beckenbach (1906-1982) and F. Wolf (1904-1989)
}

\section{EDITORS}

V. S. Varadarajan (Managing Editor)

Department of Mathematics

University of California

Los Angeles, CA 90095-1555

pacific@math.ucla.edu

Vyjayanthi Chari

Department of Mathematics

University of California

Riverside, CA 92521-0135

chari@math.ucr.edu

\section{Robert Finn}

Department of Mathematics Stanford University

Stanford, CA 94305-2125

finn@math.stanford.edu

Kefeng Liu

Department of Mathematics

University of California

Los Angeles, CA 90095-1555

liu@math.ucla.edu
Darren Long

Department of Mathematics

University of California

Santa Barbara, CA 93106-3080

long@math.ucsb.edu

Jiang-Hua Lu

Department of Mathematics

The University of Hong Kong

Pokfulam Rd., Hong Kong jhlu@maths.hku.hk

Alexander Merkurjev

Department of Mathematics

University of California

Los Angeles, CA 90095-1555

merkurev@math.ucla.edu
Sorin Popa

Department of Mathematics University of California

Los Angeles, CA 90095-1555 popa@math.ucla.edu

Jie Qing

Department of Mathematics

University of California

Santa Cruz, CA 95064

qing@cats.ucsc.edu

Jonathan Rogawski

Department of Mathematics

University of California

Los Angeles, CA 90095-1555

jonr@math.ucla.edu

\section{PRODUCTION}

pacific@math.berkeley.edu

\section{SUPPORTING INSTITUTIONS}

ACADEMIA SINICA, TAIPEI

CALIFORNIA INST. OF TECHNOLOGY INST. DE MATEMÁTICA PURA E APLICADA KEIO UNIVERSITY

MATH. SCIENCES RESEARCH INSTITUTE NEW MEXICO STATE UNIV.

OREGON STATE UNIV.

\author{
STANFORD UNIVERSITY \\ UNIV. OF BRITISH COLUMBIA \\ UNIV. OF CALIFORNIA, BERKELEY \\ UNIV. OF CALIFORNIA, DAVIS \\ UNIV. OF CALIFORNIA, LOS ANGELES \\ UNIV. OF CALIFORNIA, RIVERSIDE \\ UNIV. OF CALIFORNIA, SAN DIEGO \\ UNIV. OF CALIF., SANTA BARBARA
}

\author{
UNIV. OF CALIF., SANTA CRUZ \\ UNIV. OF MONTANA \\ UNIV. OF OREGON \\ UNIV. OF SOUTHERN CALIFORNIA \\ UNIV. OF UTAH \\ UNIV. OF WASHINGTON \\ WASHINGTON STATE UNIVERSITY
}

These supporting institutions contribute to the cost of publication of this Journal, but they are not owners or publishers and have no responsibility for its contents or policies.

See inside back cover or pacificmath.org for submission instructions.

The subscription price for 2012 is US \$420/year for the electronic version, and \$485/year for print and electronic.

Subscriptions, requests for back issues from the last three years and changes of subscribers address should be sent to Pacific Journal of Mathematics, P.O. Box 4163, Berkeley, CA 94704-0163, U.S.A. Prior back issues are obtainable from Periodicals Service Company, 11 Main Street, Germantown, NY 12526-5635. The Pacific Journal of Mathematics is indexed by Mathematical Reviews, Zentralblatt MATH, PASCAL CNRS Index, Referativnyi Zhurnal, Current Mathematical Publications and the Science Citation Index.

The Pacific Journal of Mathematics (ISSN 0030-8730) at the University of California, c/o Department of Mathematics, 969 Evans Hall, Berkeley, CA 94720-3840, is published monthly except July and August. Periodical rate postage paid at Berkeley, CA 94704, and additional mailing offices. POSTMASTER: send address changes to Pacific Journal of Mathematics, P.O. Box 4163, Berkeley, CA 94704-0163.

PJM peer review and production are managed by EditFLOW ${ }^{\mathrm{TM}}$ from Mathematical Sciences Publishers.

PUBLISHED BY PACIFIC JOURNAL OF MATHEMATICS

at the University of California, Berkeley 94720-3840

A NON-PROFIT CORPORATION

Typeset in LATEX

Copyright $(02012$ by Pacific Journal of Mathematics 


\section{PACIFIC JOURNAL OF MATHEMATICS}

Volume $257 \quad$ No. $2 \quad$ June 2012

Extending triangulations of the 2-sphere to the 3-disk preserving a

4-coloring

RUi PEDRO CARPENTIER

Orthogonal quantum group invariants of links

LIN CHEN and QINGTAO CHEN

Some properties of squeezing functions on bounded domains

FUSHENG DENG, QIAN GUAN and LIYOU ZHANG

Representations of little $q$-Schur algebras

JiE DU, QIANG FU and JiAN-PAN WANG

Renormalized weighted volume and conformal fractional Laplacians

MARÍA DEL MAR GONZÁLEZ

The $L_{4}$ norm of Littlewood polynomials derived from the Jacobi symbol 395

JONATHAN JEDWAB and KAI-UWE SCHMIDT

On a conjecture of Kaneko and Ohno

ZHONG-HUA LI

Categories of unitary representations of Banach-Lie supergroups and restriction functors

StéPhane Merigon, Karl-Hermann NeEb and Hadi

SALMASIAN

Odd Hamiltonian superalgebras and special odd Hamiltonian superalgebras of formal vector fields

Li Ren, Qiang Mu and YongZheng ZHANG

Interior derivative estimates for the Kähler-Ricci flow

Morgan SHERMAN and BEN WEINKOVE

Two-dimensional disjoint minimal graphs

LINFENG ZHOU 